\title{
AVALIAÇÃO E DESFECHO DOS PACIENTES ATENDIDOS NO SERVIÇO DE EMERGÊNCIA COM QUEIXA DE DOR TORÁCICA
}

\section{EVALUATION AND OUTCOME OF PATIENTS TREATED AT THE EMERGENCY DEPARTMENT WITH A COMPLAINT OF THORACIC PAIN}

\section{EVALUACIÓN Y ENFERMEDAD DE PACIENTES SERVIDOS EN EL SERVICIO DE EMERGENCIA CON QUEJAS DE DOLOR TORÁCICO}

\author{
Raimundo Davi Feijó Frazão ${ }^{1}$, Rita Neuma Dantas Cavalcante de Abreu², Tatiana de Medeiros Colleti \\ Cavalcante $^{3}$, Luis Rafael Leite Sampaio ${ }^{4}$
}

Como citar esse artigo: Frazão RDF, Abreu RNDC, Cavalcante TMC, Sampaio LRL. Avaliação e desfecho dos pacientes atendidos no serviço de emergência com queixa de dor torácica. Rev Enferm Atenção Saúde [Internet]. 2021 [acesso em: ]; 10(3):e202127. doi: https://doi.org/ 10.18554/reas.v10i3.4770

\section{RESUMO}

OBJETIVO: Analisar o perfil clínico de pacientes atendidos, com dor torácica, no serviço de emergência. METODOLOGIA: Pesquisa descritiva. Estudo realizado em hospital secundário do Ceará, Brasil. Foram avaliadas 110 fichas de atendimentos de pacientes atendidos por dor torácica entre os anos de 2014 e 2016. RESULTADOS: Maior prevalência de pacientes acima de 65 anos, $41(37,27 \%)$ casos. 45 (40,91\%) homens e $65(59,09 \%)$ mulheres. O único sintoma invariável, apresentado pelos pacientes que deram entrada na emergência, foi a Dor Torácica (DT) 110 (100\%) casos. Todos os pacientes 110 (100\%) foram submetidos ao Eletrocardiograma (ECG); 17 (15,45\%) foram submetidos ao teste rápido de troponina, e desse total, $05(4,54 \%)$ apresentaram resultado positivo durante o atendimento inicial. CONCLUSÃO: Do total, $92(83,65 \%)$ dos casos receberam alta hospitalar, sem qualquer intercorrência; 17 (15,45\%) foram transferidos para hospital de referência em cardiologia em Fortaleza e $01(0,90 \%)$ veio a óbito no primeiro atendimento médico.

Descritores: Cardiologia. Cuidados de enfermagem. Emergência.

\footnotetext{
1 Graduado em Enfermagem, Especialização em Enfermagem em Terapia Intensiva da Universidade de Fortaleza/UNIFOR, Fortaleza CE, Brasilhttp://orcid.org/0000-0002-1962-7175

2 Enfermeira do Instituto Dr. José Frota. Doutora em Biotecnologia da Rede Nordeste de Biotecnologia (RENORBIO-UFC). Mestre em Cuidados Clínicos em Saúde da Universidade Estadual do Ceará (UECE). Docente da Universidade de Fortaleza (UNIFOR). Docente do Mestrado em Tecnologia e Inovação em Enfermagem da Universidade de Fortaleza. http://orcid.org/0000-0002-5130-0703

3 Doutora em Enfermagem, Universidade Federal de São Paulo/UNIFESP, Docente da Universidade de Fortaleza/UNIFOR e coordenadora da Especialização em Enfermagem em Terapia Intensiva/UNIFOR, Fortaleza CE, Brasilhttp://orcid.org/0000-0002-6564-9746

${ }^{4}$ Docente da URCA. Doutor em Farmacologia, Universidade Federal do Ceará/UFC, Fortaleza CE, Brasil. Docente do Mestrado em Tecnologia e Inovação em Enfermagem da Universidade de Fortaleza.
} 


\begin{abstract}
OBJECTIVE: To analyze the clinical profile of patients treated with chest pain in the emergency department. METHOD: Descriptive research. Study performed in a secondary hospital in Ceará, Brazil. A total of 110 patient charts were evaluated for patients treated for chest pain between 2014 and 2016. RESULTS: Higher prevalence of patients over 65 years $41(37.27 \%)$ cases. $45(40.91 \%)$ men and $65(59.09 \%)$ women. The only invariable symptom presented by patients entering the emergency room was thoracic pain (DT) 110 (100\%) cases. All $110(100 \%)$ patients underwent electrocardiogram (ECG); $17(15,45 \%)$ underwent the rapid troponin test, and of that total. CONCLUSION: Of the total, $92(83.65 \%)$ of the cases were discharged without any complications; $17(15.45 \%)$ were transferred to a referral hospital in cardiology in Fortaleza, and $01(0.90 \%)$ died at the first medical visit.
\end{abstract}

Descriptors: Cardiology. Nursing care. Emergency.

\title{
RESUMEN
}

OBJETIVO: Analizar el perfil clínico de pacientes tratados con dolor torácico en urgencias. METODOLOGÍA: Investigación descriptiva. Estudio realizado en un hospital secundario de Ceará, Brasil. Se evaluaron 110 registros de atención de pacientes tratados por dolor torácico entre los años 2014 y 2016. RESULTADOS: Mayor prevalencia de pacientes mayores de 65 años $41(37,27 \%)$ casos. 45 (40.91\%) hombres y $65(59.09 \%)$ mujeres. El único síntoma invariable presentado por los pacientes que acudieron a la sala de emergencias fue el dolor de pecho (TD) 110 (100\%) casos. Todos los pacientes 110 (100\%) se sometieron a un electrocardiograma $(\mathrm{ECG}) ; 17(15,45 \%)$ se sometieron a la prueba rápida de troponina, y de este total, $05(4,54 \%)$ tuvieron un resultado positivo durante la atención inicial. CONCLUSIÓN: Del total, $92(83,65 \%)$ de los casos fueron dados de alta del hospital sin complicaciones; $17(15,45 \%)$ fueron transferidos a un hospital de referencia en cardiología en Fortaleza y $01(0,90 \%)$ murieron en la primera atención médica.

Descriptores: Cardiología. Cuidado de enfermera. Emergencia.

\section{INTRODUÇÃO}

A população mundial está envelhecendo. Nas últimas décadas, observou-se em grande parte do mundo o aumento significativo do número de idosos. Entre 2000 e 2030, estima-se que a porcentagem de idosos com mais de 65 anos deverá passar de $6,9 \%$ para $12 \% .^{1}$ Entre as causas de morte e incapacidade no Brasil e no mundo, as Doenças Cardiovasculares (DCVs) ganham espaço. ${ }^{2}$ Dentre estas, a Síndrome coronariana aguda é um dos principais problemas de saúde pública, e a busca por intervenções que apresentem benefício comprovado na redução da incidência dessa doença e de suas complicações passa a ser prioridade, principalmente para a população idosa como grupo etário em rápido crescimento no Brasil e no mundo. ${ }^{3}$

No Brasil, o Serviço de Acolhimento e Classificação de Risco (SACR) é feito por meio de protocolos e deve ser executado por enfermeiros, através da resolução do Conselho Federal de Enfermagem (COFEN) 661/2021, sendo 
uma atividade que demanda competência técnica e científica. ${ }^{4}$

Um dos sintomas referidos na emergência é a Dor Torácica (DT). Essa queixa torna-se um sintoma de alarme na emergência e relaciona-se a fatores sensoriais, comportamentais, cognitivos e socioculturais, além da doença de base. Sendo, portanto, multifatorial. ${ }^{5}$

A Unidade Hospitalar (UH) onde foi realizado o estudo caracteriza-se como Hospital de Pequeno Porte (HPP), não sendo referência clínica, ou cirúrgica, a nenhuma especialidade médica. Os pacientes graves atendidos são estabilizados e referenciados a hospitais terciários da capital cearense. Portanto, viu-se necessário traçar um perfil dessa população e, posteriormente, ser implementado um plano sistemático que vise a melhora da qualidade assistencial.

Objetivou-se analisar o perfil clínico e desfecho de pacientes com dor torácica no serviço de emergência.

\section{METODOLOGIA}

Pesquisa descritiva com abordagem quantitativa, retrospectiva. $\mathrm{O}$ estudo foi realizado em um hospital secundário da rede SUS, localizado em um município do Estado do Ceará, Brasil. A população foi integrada de 150 fichas, de atendimento, daqueles que apresentaram dor torácica na emergência entre os anos de 2014 a 2016.
No entanto, 110 foi o número total de casos avaliados, segundo os critérios estabelecidos.

A coleta de dados foi realizada nos meses de junho e julho de 2017 após aprovação do Projeto de Pesquisa pelo Comitê de Ética. A amostra foi constituída de fichas de atendimentos e analisados dados secundários: sinais vitais, exames realizados, queixa principal, conduta final.

Como critérios de inclusão, foram analisadas fichas de pacientes maiores de 18 anos e com registros completos do atendimento. Como critérios de exclusão, não foram computadas informações contidas em fichas de pacientes menores de 18 anos, ou que possuíam informações incompletas referentes ao atendimento. Assim, do total de 150 atendimentos, 110 casos foram avaliados.

A análise de dados se deu de forma descritiva, sendo realizada por meio de frequências absolutas e relativas. Sobre as variáveis, foram vistos: exames e laudo, idade, gênero, queixa principal, diabetes mellitus, hipertensão arterial, obesidade, pressão arterial, frequência cardíaca, frequência respiratória, temperatura, saturação de oxigênio, características da dor, terapêutica medicamentosa utilizada, desfecho final.

O projeto foi aprovado pelo Comitê de Ética em Pesquisa em Seres Humanos COÉTICA/UNIFOR da Universidade de 
Fortaleza com o Parecer N. 2.011.067. Por se tratar de uma pesquisa documental, foi realizado o Termo de Fiel Depositário.

\section{RESULTADOS}

Os dados mostraram que a idade acima de 65 anos representou 41 (37,27\%) dos casos, e gênero feminino com 65 $(59,09 \%)$. Ou seja, um número superior de pacientes femininos apresentou sintomatologia típica, porém, todos os 110 (100\%) referiram DT e foram avaliados para SCA.

Tabela 1: Caracterização clínica dos pacientes com dor torácica atendidos na emergência.

\begin{tabular}{c|c|c|c}
\hline VARIÁVEL & FUNDAMENTAÇÃO & N & $\%$ \\
\hline Idade Acima de 65 Anos & População Prevalente & 41 & 37,27 \\
\hline Sexo Feminino & Gênero Prevalente & 65 & 59,09 \\
\hline Dor Torácica & Queixa Principal & 110 & 100 \\
\hline Realizou Eletrocardiograma & Submissão ao Exame & 110 & 100 \\
\hline ECG Sem Alterações & ECG Convencional & 62 & 56,36 \\
\hline Diabetes Mellitus & Diagnóstico Prévio & 09 & 9,9 \\
\hline Valores Pressóricos & Igual ou inferior à 120x80 mmHg & 48 & 43,63 \\
\hline Frequência Cardíaca & $60-100$ bpm & 70 & 63,63 \\
\hline Frequência Respiratória & $12-20$ rpm & 83 & 75,45 \\
\hline Saturação de Oxigênio & $<94 \%$ & 16 & 14,54 \\
\hline Temperatura Corporal & $>37,8^{\circ} \mathrm{C}$ & 09 & 8,18 \\
\hline Características da Dor & Dor em Aperto & 68 & 61,81 \\
Torácica & & & 17 \\
\hline Teste de Troponina & Entre os resultados ( - ) e ( ) & 07 & 6,36 \\
\hline Realizou Raio X & Tórax & 43 & 39,09 \\
\hline Uso de Ácido Acetilsalicílico & AAS & 12 & 10,90 \\
\hline Antagonistas ADP & Clopidogrel & 25 & 22,72 \\
\hline Nitratos & Dinitrato de Isossorbida & 83,65 \\
\hline Alta Hospitalar & Após Melhora Clínica & 15,45 \\
\hline Transferências & Hospital de Referência & 01 & 0,90 \\
\hline Óbitos & Na Unidade Hospitalar & 17 &
\end{tabular}

Em relação a realização do Eletrocardiograma (ECG), esse fato foi uma constante. Todos os pacientes 110 (100\%) foram submetidos e visto que, a maioria, ou seja, $62(56,36 \%)$ apresentou esse exame sem alterações no primeiro atendimento, segundo registros médicos.

Em relação as DCVs e metabólicas prévias, por exemplo, o DM esteve registrado em 09 (9,9\%), a HAS foi registrada em $61(67,1 \%)$ casos e a obesidade foi registrada em $18(19,8 \%)$ dos casos.

Referente os valores pressóricos encontrados nos registros no momento da queixa sintomática, $48 \quad(43,63 \%)$ apresentaram Pressão Arterial (PA) igual ou inferior a 120x80 mmHg.

Sobre a frequência cardíaca (FC), foi visto que $70(63,63 \%)$ revelaram valores entre 60 a $100 \mathrm{bpm}$. Do total, 03 $(2,72 \%)$ tiveram uma FC abaixo de 60 
bpm. Outros $37(33,63 \%)$ apresentaram FC acima de 100 bpm.

Em relação a Frequência Respiratória (FR), $83 \quad(75,45 \%)$ apresentaram-se na emergência com FR maior que 20 incursões por minuto. Relativo à Saturação de Oxigênio (SPO2), $16(14,54 \%)$ apresentaram SPO2 <94\% durante $\mathrm{o}$ atendimento inicial, medida avaliada por meio do oxímetro de pulso. Vale ressaltar que a $\mathrm{UH}$ onde se deu a pesquisa não dispunha de gasometria. A temperatura corporal, também avaliada, apresentou $09(8,18 \%)$ com medidas > $37,8^{\circ} \mathrm{C}$.

As características da DT foram classificadas de acordo com a queixa. Estava registrado que $29 \quad(26,36 \%)$ referiram dor em queimação, 68 (61,81\%) dor em aperto, $08(7,27 \%)$ relataram dor em pontada, 05 (4,54\%) dor em opressão. Sobre a troponina, foram vistos que 17 $(15,45 \%)$ foram submetidos ao teste rápido, e desse total, 05 (4,54\%) apresentaram resultado positivo durante o atendimento inicial. Outro exame solicitado pelo médico, em alguns casos, foi a radiografia do tórax, 07 (6,36\%).

Relacionado a terapêutica medicamentosa prescrita pelo médico, observou-se que $43(39,09 \%)$ fizeram uso de AAS, 25 (22,72\%) beneficiaram-se do dinitrato de isossorbida (isordil), 06 $(5,45 \%)$ usaram betabloqueadores, em especial o propranolol, $07 \quad(6,36 \%)$ utilizaram opióides, em destaque, a morfina. Outros fármacos tiveram também destaque clínico, por exemplo, o clopidogrel, esse foi inserido em 12 $(10,90 \%)$ dos atendimentos.

Nesse estudo, a maior parte dos indivíduos participantes teve desfecho satisfatório, ou seja, $92(83,65 \%)$ dos mesmos receberam alta hospitalar, 17 $(15,45 \%)$ foram transferidos para hospital de referência em cardiologia e 01 (0,90\%) veio a óbito.

\section{DISCUSSÃO}

No presente estudo, houve maior número de mulheres atendidas na emergência. Até 65 anos a DAC é mais prevalente nos homens, mas a partir dos 80 anos sua prevalência é equivalente em ambos os sexos ${ }^{6}$. Sobre os sintomas, a DT foi referida em todos os casos. O ECG foi realizado em todos os pacientes desse estudo.

Foi visto que prevaleceu resultados normais do ECG no atendimento inicial, segundo registro médico. Sobre isso, os protocolos alertam que esse fato não exclui o risco de SCA. ${ }^{7}$

Em relação às DCVs e metabólicas, por exemplo, a Hipertensão Arterial Sistêmica (HAS), o DM e a obesidade são agravantes abordados em estudo realizado em Porto Alegre. ${ }^{8}$ As cifras pressóricas 
destacam valores de PA igual, ou inferior, a 120x80 mmHg. Vale lembrar que o objetivo desse estudo não foi triar pacientes que poderiam ter HAS.

A FC foi uma variável em que, a maior parte dos pacientes, também apresentou valores normais. Salienta-se que esses sinais clínicos no contexto da SCA podem ter implicações importantes. ${ }^{9}$ A FR aumentada foi mais prevalente nesse estudo, ou seja, com FR maior que 20 rpm. Pacientes com taquipneia e com sintomatologia clínica de SCA podem estar apenas ansiosos ${ }^{10}$, ou estar evoluindo para complicações, por exemplo, SCA, edema agudo de pulmão, dentre outros. ${ }^{9}$ Outro estudo mostrou que menos de $20 \%$ apresentou alterações importantes no exame físico inicial, a presença de taquipneia, hipotensão, e estertores pulmonares, dentre outros, devem chamar a atenção, pois podem indicar grande comprometimento miocárdico, com potencial para falência cardíaca aguda. ${ }^{11}$

Sobre os níveis de oxigênio mensurados através do oxímetro de pulso, a maioria revelou cifras $\mathrm{SPO} 2>94 \%$. No entanto, vale ressaltar que a $\mathrm{UH}$, onde se deu a pesquisa, não dispunha de gasometria. Nesse sentido, salienta-se que $\mathrm{O}_{2}$ administrado desnecessariamente pode levar a vasoconstrição, aumento da resistência vascular sistêmica e da PA, sendo assim, prejudicial. ${ }^{12}$ Em relação a temperatura corporal, prevaleceu os pacientes com temperatura $<37,8^{\circ} \mathrm{C}$.

Sobre as características da DT, predominou a queixa de dor em aperto. Outro estudo semelhante, mostrou que a característica mais prevalente foi a dor em queimação $12(52,17 \%)$, o que mostra uma heterogeneidade sintomática. $^{13}$ Sobre a troponina, foram vistos que um pequeno percentual foi submetido ao teste rápido. Sendo que a sua elevação aumenta o risco de morte e re(infarto) nos primeiros 6 meses, quando comparado aos pacientes troponina negativos. ${ }^{14,15}$ É importante ressaltar, que nenhum dos pacientes com cifras negativas foi novamente submetido ao exame, após o atendimento inicial. Sabe-se que as troponinas na admissão estão dentro do valor de normalidade em até um quinto dos casos que depois confirmam diagnóstico de SCA. ${ }^{16}$ Ou seja, não foi seguido um protocolo especifico para esse recurso.

Em relação ao Raios $X$ (tórax), houve baixo percentual desse exame de imagem, isso pode ser atribuído, por exemplo, a não disponibilidade e/ou a distância mais próxima de uma UH que dispunha desse método.

Os fármacos relacionados a abordagem das SCAs foram o AAS, os nitratos, betabloqueadores em especial o propranolol, dentre outros. Essas condutas podem ser passíveis de mudanças nos mais 
variados contextos. O AAS, por exemplo, é importante na SCA, seu uso implica redução da mortalidade. ${ }^{17}$ Apesar disso, pesquisas revelam fenômenos isquêmicos mesmo com uso contínuo ${ }^{18}$, mas continua sendo o antiplaquetário de eleição na SCA, como mostra o estudo ISIS-2 (Second International Study of Infarct Survival), reduzindo a mortalidade em $23 \%$, isoladamente, quase tanto quanto a Estreptoquinase (SK). Os medicamentos morfina e clopidogrel também foram citados em menor escala e possuem efeitos importantes na SCA. ${ }^{19}$

Nesse estudo, a maior parte dos indivíduos teve desfecho satisfatório do ponto de vista clínico, ou seja, receberam alta hospitalar sem qualquer intercorrência grave quando descartado um quadro de SCA. 17 foram transferidos para hospital de referência em cardiologia e 01 veio a óbito no hospital do primeiro atendimento médico.

Os dados reforçam a importância do preparo dos profissionais de saúde para a avaliação criteriosa dos pacientes que procuram atendimento no serviço de emergência. Cabe ressaltar que estudos sobre o conhecimento dos dados sociodemográficos e clínicos podem favorecer no planejamento e efetivação da assistência, implicando melhorias na qualidade da mesma. ${ }^{20}$ Assim, a dor, como um dos sinais vitais, precisa sempre ser Rev Enferm Atenção Saúde [Online]. Out/Dez 2021; 10(3):e202127 valorizada pela equipe de enfermagem e demais profissionais.

\section{CONCLUSÃO}

Diante do exposto, o objetivo de avaliar o perfil clínico e desfecho dos pacientes com dor torácica atendidos na emergência de um hospital do interior do estado do Ceará foi atingido com as conclusões a saber:

O perfil clínico dos pacientes atendidos na emergência, por dor torácica, foi de idade superior a 65 anos, todos os casos referiram dor de origem torácica com prevalência de dor em aperto, toda a amostra foi submetida ao ECG, onde a maioria apresentou resultado inalterado. Referente a frequência cardíaca, a maior parcela revelou valores dentro da normalidade. Maiores percentuais apresentaram taquipneia no primeiro atendimento. Parcela significativa dessa amostra foi submetida ao teste rápido de troponina, porém, a oferta do mesmo não foi abrangente a todos os pacientes que tinham indicação a esse exame por razão da deficiência quantitativa desse insumo. Nesse contexto, alto percentual não realizou radiografia de tórax. Os fármacos prescritos pelo médico foram o AAS, o clopidogrel, o dinitrato de isossorbida, dentre outros. 
Quanto ao desfecho desses indivíduos, 92 pessoas receberam alta hospitalar sem qualquer intercorrência grave, 17 foram transferidos e um paciente veio à óbito no hospital do primeiro atendimento médico.

Desse modo, obteve-se um perfil clínico e desfecho final desses pacientes que se apresentaram com DT na emergência. Nesse sentido, é possível conhecer melhor essa clientela e obter subsídios para a construção posterior de uma estratégia sistemática de atendimento individualizado à essa população. Não menos importante criar uma discussão sobre a importância de uma educação continuada para os profissionais de saúde bem como a persuasão do núcleo gestor hospitalar em relação a maiores investimentos no setor.

\section{REFERÊNCIAS}

1. Lapa E. Doença Coronariana na População Idosa. In: Diniz LR, Gomes DCDA, Kitner D. Geriatria. 1 ed. Rio de Janeiro: Medbook, 2019. p.216-219.

2. Schmidt MI, Duncan BB, Silva GA, Menezes AM, Monteiro CA, Barreto SM, et al. Doenças crônicas não transmissíveis no Brasil: carga e desafios atuais [Internet]. [S.1.]: The Lancet; 2011 [citado em 23 dez 2021]; 14 p. (Saúde no Brasil, 4). Disponível em: https://www.idec.org.br/pdf/schmid tetal_lancet2011.pdf

3. França KM. Abordagem das Síndromes coronarianas agudas em idosos em hospital geral do SUS. [Internet]. Santos (São Paulo). Dissertação [Mestrado em Saúde Coletiva] - Universidade Católica de Santos;2014 [citado em $23 \mathrm{dez}$ 2021]; 104p. Disponível em: https://tede.unisantos.br/bitstream/t ede/901/2/Klauber\%20M.\%20de\% 20Fran\%C3\%A7a.pdf

4. Conselho Federal de Enfermagem (Brasil). Resolução n. ${ }^{\circ}$ 661, de 15 de fevereiro de 2021. Atualiza e normatiza, no âmbito do Sistema Cofen/Conselhos Regionais de Enfermagem, a participação da equipe de enfermagem na atividade de classificação de riscos [Internet] Brasília, DF: COFEN; 2021 [citado em 23 dez 2021]. Disponível em: http://www.cofen.gov.br/resolucaocofen-no-661-2021_85839.html

5. Jacob J. Avaliação e controle da dor em crianças. In: Hockenberry MJ, Wilson D. Wong: fundamentos de enfermagem pediátrica. 8ed. Rio de Janeiro: Mosby; 2011. p. 162-202.

6. Keller NM, Feit F. Coronary artery disease in the geriatric population. Prog Cardiovasc Dis. [Internet]. 1996 Mar/Apr [citado em $23 \mathrm{dez}$ 2021]; 38(5):407-18. Disponível em:

https://www.sciencedirect.com/scie nce/article/pii/S0033062096800351 /pdf?md5=1f4c906196b8ba13428b 151cd723f976\&pid=1-s2.0S0033062096800351-main.pdf

7. Diretrizes da Sociedade Brasileira de Cardiologia sobre angina instável e infarto agudo do miocárdio sem supradesnível do segmento ST: II Edição, 2007, atualização 2013/2014. Arq Bras Cardiol. [Internet]. mar 2014 [citado em 23 dez 2021]; 102(3 Supl 1):1-46. Disponível em: http://publicacoes.cardiol.br/consen so/2014/Diretriz_de_IAM.pdf

8. Lemos KF, Davis R, Moraes MA, Azzolin K. Prevalência de fatores 
de risco para síndrome coronariana aguda em pacientes atendidos em uma emergência. Rev Gaúch Enferm. [Internet]. mar 2010 [citado em $23 \mathrm{dez} 2021$ ]; 31(1): 129-35. Disponível em: https://www.scielo.br/j/rgenf/a/cFC k8KMt6BYKWWmy3KPCgFy/?fo rmat $=$ pdf\&lang $=\mathrm{pt}$

9. Herck JLV, Claeys MJ, Paep R, Herck PLV, Vrints CJ, Jorens PG. Management of cardiogenic shock complicating acute myocardial infarction. Eur Heart J Acute Cardiovasc Care [Internet]. 2015 Jun [citado em 23 dez 2021]; 4(3):278-97. Disponível em: https://academic.oup.com/ehjacc/ar ticlepdf/4/3/278/34170860/ehjacc0 278.pdf

10. Bangalore S, Makani H, Radford M, Thakur K, Toklu B, Katz SD, et al. Clinical outcomes with betablockers for myocardial infarction: a meta-analysis of randomized trials. Am J Med. [Internet]. 2014 Oct [citado em 23 dez 2021]; 127(10):939-53. Disponível em: https://www.sciencedirect.com/scie nce/article/pii/S0002934314004707 /pdfft?md5=dbaac32017cd10a2ce7 22a81a54c6f95\&pid=1-s2.0S0002934314004707-main.pdf

11. Piegas LS, Timerman A, Feitosa GS, Nicolau JC, Mattos LAP, Andrade MD, et al. V diretriz da Sociedade Brasileira de Cardiologia sobre tratamento do infarto agudo do miocárdio com supradesnível do segmento ST. Arqu Bras Cardiol. [Internet]. ago 2015 [citado em 23 dez 2021]; 105(2 supl 1):1-81.

Disponível em: http://publicacoes.cardiol.br/2014/d iretrizes/2015/02_TRATAMENTO $\% 20 D O \% 20 I A M \% 20 \mathrm{COM} \% 20 \mathrm{SU}$ PRADESNIVEL\%20DO\%20SEG MENTO\%20ST.pdf
12. Timerman A, Feitosa GA Síndromes coronárias agudas. Rio de Janeiro: Atheneu; 2003.

13. Santos F; Freire PB; Ribeiro JA. Abordagem da dor torácica pelo enfermeiro em uma unidade de pronto atendimento na visão do paciente. Enferm Rev. [Internet]. maio/ago 2016 [citado em $23 \mathrm{dez}$ 2021]; 19(2):199-211. Disponível em:

http://periodicos.pucminas.br/index .php/enfermagemrevista/article/vie w/13149/10300

14. Kavsak PA, Newman AM, Lustig V, MacRae AR, Palomaki GE, Ko DT, et al. Long-term health outcomes associated with detectable troponin I concentrations. Clin Chem. [Internet]. $2007 \mathrm{Feb}$ [citado em 23 dez 2021]; 53(2):220-7. Disponível em:

https://academic.oup.com/clinchem /articlepdf/53/2/220/32689220/clin chem0220.pdf

15. Heidenreich PA, Alloggiamento T, Melsop K, McDonald KM, Go AS, Hlatky MA. The prognostic value of troponin in patients with non-ST elevation acute coronary syndromes: a meta-analysis. J Am Coll Cardiol. [Internet]. 2001 [citado em 04 jun 2020]; 38(2):47885. Disponível em:

https://www.sciencedirect.com/scie nce/article/pii/S0735109701013882 /pdfft?md5=af78da1e891 cc8e0a45 82b3b18774efd\&pid=1-s2.0S0735109701013882-main.pdf

16. Hoeller RG, Gimenez MR, Reichlin $\mathrm{T}$, Twerenbold R, Zellweger C, Moehring B, et al. Normal presenting levels of high-sensitivity troponin and myocardial infarction. Heart. [Internet]. 2013 [citado em 04 jun 2020]; 99(21):1567-72. Disponível em: https://heart.bmj.com/content/heartj n1/99/21/1567.full.pdf 
17. Martins, HS. Síndromes coronarianas agudas sem elevação do segmento ST. In: Martins HS, Brandão Neto RA, Velasco IT. Medicina de Emergência. 12. ed. Barueri (SP): Manole; 2017. p. 925-972.

18. Muir AR, McMullin MF, Patterson C, McKeown PP. Assessment of aspirin resistance varies on a temporal basis in patients with ischaemic heart disease. Heart [Internet]. 2009 Aug [citado em 23 dez 2021]; 95(15):1225-229.

Disponível em; https://www.ncbi.nlm.nih.gov/pmc/ articles/PMC2705011/pdf/HRT-9515-1225.pdf

19. Roffi M, Patrono C, Collet JP, Mueller C, Valgimigli M, Andreotti F, et al. 2015 ESC Guidelines for the management of acute coronary syndromes in patients presenting without persistent ST-segment elevation: task force for the management of acute coronary

RECEBIDO: $11 / 07 / 2020$

APROVADO: 04/08/2021

PUBLICADO: dez/2021 syndromes in patients presenting without persistent ST-Segment Elevation of the European Society of Cardiology (ESC). Eur Heart J. [Internet]. 2016 Jan [citado em 23 dez 2021]; 37(3):267-315.

Disponível em:

https://academic.oup.com/eurheartj/ articlepdf/37/3/267/23493022/ehv320.pdf

20. Soares DS, Santos TS, Maier SRO, Sudré MRS, Flores CAS, Oliveira WS. Caracterização das vítimas de infarto do miocárdio admitidas em uma unidade coronariana. Rev Enferm Atenção Saúde [Internet]. ago/dez 2019 [citado em $23 \mathrm{dez}$ 2021]; 8(2):98-106. Disponível em: http://seer.uftm.edu.br/revistaeletro nica/index.php/enfer/article/view/3 $762 /$ pdf 